\title{
O Atendimento Compartilhado na Perspectiva da Atuação Multiprofissional na Atenção Primária à Saúde
}

\author{
Caroline Maria Franke ${ }^{1}$, Valéria Baccarin laniski², Linda Cristina Sangoi Haas ${ }^{3}$
}

\begin{abstract}
RESUMO
Sob a lógica do Sistema Único de Saúde (SUS), as consultas compartilhadas, realizadas por profissionais de diferentes áreas, são utilizadas como ferramenta de promoção da saúde e prevenção de doenças, bem como com o objetivo de aumentar o cuidado e dar autonomia aos sujeitos nas tomadas de decisão sobre sua própria saúde. As residências multiprofissionais auxiliam nesse processo, apresentando um maior número de profissionais e qualificando-os para o cuidado integral ao usuário na Atenção Básica. Este artigo caracteriza-se como um relato de experiência sobre a realização de consultas compartilhadas executadas por profissionais de saúde residentes nas áreas de nutrição e educação física, além de ressaltar a importância do trabalho multiprofissional realizado em uma Unidade Básica de Saúde da Família de um município do Noroeste do Estado do Rio Grande do Sul. Os educadores físicos e nutricionistas trabalham de maneira semelhante, visando o cuidado sem o uso de tratamentos farmacológicos, auxiliando no processo de promoção e recuperação da saúde e na prevenção de doenças por meio do estímulo à adesão de hábitos de vida saudáveis, com atenção especial para a adoção de uma alimentação saudável e da prática regular de atividades físicas. Nessa lógica ampliada do cuidado, os profissionais de saúde e usuários são responsabilizados de igual maneira pelo processo de cuidado, sendo o usuário o protagonista da atenção à saúde.
\end{abstract}

Palavras-chave: Atenção Primária à Saúde. Equipe interdisciplinar de saúde. Saúde pública.

\section{CALL SHARED IN PERSPECTIVE IN ACTING MULTIPROFISSIONAL PRIMARY HEALTH CARE}

\begin{abstract}
Under the logic of the Single Health System (SUS), shared consultations carried out by professionals from different areas are used as a tool for health promotion and disease prevention, as well as for the purpose of increasing care and giving autonomy to the subjects to make decisions about their own health. The multiprofessional residences help in this process, having a greater number of professionals and qualifying them for the integral care to the user in the Primary Care. This article is an experience report about the realization of shared consultations carried out by resident health professionals in the areas of nutrition and physical education, as well as highlighting the importance of multiprofessional work carried out in a Basic Family Health Unit of one municipality of the Northwest region of the state of Rio Grande do Sul. Physical educators and nutritionists work in a similar way, aiming at the care without the use of pharmacological treatments, aiding in the process of promotion and recovery of health and in the prevention of diseases through stimulation adherence to healthy lifestyle habits, with special attention to the adoption of healthy eating and regular practice of physical activities. In this expanded logic of care, health professionals and users are held accountable for the care process, and the user is the protagonist of health care. Keywords: Primary Health Care. Interdisciplinary health team. Public health.
\end{abstract}

Recebido em: 9/7/2017

Aceito em: 14/11/2018

\footnotetext{
Bacharel em Educação Física (Universidade Federal de Santa Maria - UFSM). Especialista em Saúde da Família pelo Programa de Residência Multiprofissional em Saúde da Família Unijuí/Fumssar. carolinemariafranke@hotmail.com

2 Graduação em Nutrição (Universidade Regional do Noroeste do Estado do Rio Grande do Sul - Unijuí, 2014). Especialista em Saúde da Família pelo Programa de Residência Multiprofissional em Saúde da Família Unijuí/Fumssar (2017). Aluna do curso de Mestrado do Programa de Pós-Graduação em Gerontologia Biomédica (Geronbio) - Escola de Medicina da Pontifícia Universidade Católica do Rio Grande do Sul (PUCRS, 2017-2019). Membro do Grupo de Interesse em Saúde Pública e Envelhecimento (Gispe) da Sociedade Brasileira de Geriatria e Gerontologia (SBGG/RS), do Grupo de Estudos em Risco Cardiometabólico, Envelhecimento e Nutrição (Gericen) e do Grupo de Pesquisa e Extensão em Atenção Multiprofissional ao Longevo (Ampal). valeriaianiski@yahoo.com.br

3 Graduação em Enfermagem (Universidade Federal de Santa Maria - UFSM, 1983). Especialização em Saúde Pública pela Unaerp (1992). Enfermeira da Fundação Municipal de Saúde de Santa Rosa, tutora de Área da Residência Multidisciplinar Unijuí/Fumssar e orientadora da Especialização em Saúde da Família da UFPel. lindacristinasangoihaas@yahoo.com.br
} 


\section{INTRODUÇÃO}

Nas últimas décadas vários programas foram propostos na área da saúde, com o objetivo de contribuir para transformar o Sistema Único de Saúde (SUS). Entre eles, temos o Programa de Saúde da Família (PSF), criado em 1994, e, posteriormente, modificado para Estratégia da Saúde da Família (ESF), com a perspectiva de fortalecer a atenção básica e ser capaz de resolver até $85 \%$ dos problemas de saúde da população (VIEIRA et al., 2016). Mais recentemente, em 2008, surgiram os Núcleos de Apoio à Saúde da Família (Nasf), que se configuram como um serviço de suporte especializado para as equipes, e que são acionados de acordo com as necessidades da ESF no sentido de aumentar a resolutividade das ações na atenção básica por intermédio do apoio matricial.

Com vistas ao empoderamento e fortalecimento da Atenção Primária à Saúde (APS), em 2006 tivemos a publicação da Política Nacional de Atenção Básica (PNAB), que foi, mais tarde, reeditada (no ano de 2011), a qual estabelece as diretrizes e normas para a organização da Atenção Básica, ESF e o Programa de Agentes Comunitários de Saúde (TOMASI; RIZZOTTO, 2013; BRASIL, 2010).

Na busca de romper com paradigmas preestabelecidos e o modelo biomédico, a ESF incorpora um novo pensar e agir no sentido de mudança e conversão do modelo assistencial. Na perspectiva da integralidade do cuidado e do usuário como protagonista das ações em saúde, o SUS tem estimulado a presença ativa dos sujeitos e as interações subjetivas e dinâmicas entre usuários e profissionais, exigindo ampliação dos horizontes da racionalidade que orientam as tecnologias do cuidado e os agentes de práticas no cuidado em saúde (ALMEIDA et al., 2015; SOUZA et al., 2008).

Vislumbrando uma assistência integral e humanizada, buscou-se o estabelecimento de equipes multiprofissionais. $O$ trabalho multiprofissional tem como objetivo fortalecer a dinâmica da resolutividade e de uma assistência holística. Neste contexto, as residências multiprofissionais apresentam-se firmadas em um cenário de mudança de cuidados em saúde, em que aspectos, como a promoção da saúde, a descentralização do cuidado e o trabalho multiprofissional e interdisciplinar, são agregados em torno do saber fazer no campo da saúde, na perspectiva de contribuir para a integralidade do cuidado ofertado aos indivíduos de acordo com as realidades loco-regionais (CORRÊA et al., 2014).
Em todos os níveis de atenção à saúde percebe-se a necessidade do trabalho interdisciplinar e multiprofissional, para que a equipe de saúde possa desenvolver um olhar mais amplo, com vistas às subjetividades de cada usuário na busca pelo fim do modelo assistencial biomédico, possibilitando abordagens múltiplas e intervenções técnicas e de interação dos usuários com diferentes profissões (COSTA et al., 2014).

Na transição etária e epidemiológica vivenciada atualmente, uma das maiores preocupações em saúde são as Doenças Cônicas Não Transmissíveis (DCNTs). Nas últimas décadas, as DCNTs passaram a ser responsáveis pelas principais causas de morte no Brasil, com destaque para as doenças cardiovasculares (DCVs), diabetes mellittus (DM), síndrome metabólica, neoplasias, hipertensão arterial sistêmica (HAS) e dislipidemia (BRASIL, 2008).

Visando a uma assistência centrada no protagonismo dos usuários, na corresponsabilidade, na não medicalização e no apoio multiprofissional, o atendimento compartilhado é uma das ações que podem ser desenvolvidas pelos profissionais do Nasf, corroborando para uma intervenção multiprofissional e interdisciplinar, de ajuda mútua, na perspectiva da troca de experiência entre profissionais e entre profissionais e usuários, para a promoção de uma assistência ampla e resolutiva (BRASIL, 2009).

Diante do exposto, o presente artigo objetiva descrever a importância da atuação multiprofissional na APS, por meio da realização de atendimentos compartilhados por profissionais de saúde residentes das áreas de nutrição e educação física em município da Região Noroeste do Estado do Rio Grande do Sul.

\section{MÉTODO}

Trata-se de um relato de experiência acerca da realização de atendimentos compartilhados por profissionais de saúde residentes das áreas de nutrição e educação física, bem como descrever a importância da atuação multiprofissional na APS.

As consultas compartilhadas foram organizadas semanalmente nos terceiros turnos, que são horários de atendimento clínicos e/ou de realização de ações grupais instituídas a partir do Programa de Residência Multiprofissional. Estes momentos decorrem das 18 horas e 30 minutos até as 21 horas e 30 minutos, nas segundas-feiras, com a finalidade de promover acesso assistencial aos usuários com DCNTs que não conseguem acessar o serviço de saúde durante o período da manhã e/ou da tarde. 
As consultas compartilhadas baseiam-se em atendimentos em saúde realizados por profissionais de diferentes áreas para construir, conjuntamente com o usuário, uma proposta de adesão às demandas em saúde, de forma que esta reflita as condições socioeconômicas, culturais e as rotinas dos sujeitos. Essa metodologia está relacionada com os pressupostos da clínica ampliada, assumindo que profissionais de saúde possam atuar concomitantemente ante a complexidade das demandas e manejo de diagnósticos, fazendo-os refletir e compartilhar a gestão de tais situações com usuários e famílias, reconhecendo a singularidade de cada situação (BRASIL, 2010).

Foram respeitados todos os preceitos éticos que regem uma pesquisa com seres humanos, de acordo com a Resolução no 466/2012 do Conselho Nacional de Saúde/Ministério da Saúde, legislação brasileira vigente.

\section{RESULTADOS E DISCUSSÕES}

A transição etária e epidemiológica exige do sistema de saúde atual ações integrais, resolutivas e que promovam a qualidade de vida com minimização de sintomas, agravos e dissociação de doenças. As DCNTs são um dos principais problemas de saúde pública do país, pois, quando não acompanhadas e tratadas adequadamente, somam-se a outros determinantes e condicionantes do adoecimento, interferindo na saúde da população, gerando despesas milionárias aos cofres públicos com tratamentos medicamentosos e demais ações para a manutenção da vida (COSTA et al., 2014; ARPINI; ARPINI, 2014; BRASIL, 2008).

Destaca-se que as DCNTs são responsáveis pela maior parte de mortes prematuras e perda da qualidade de vida, sendo consideradas uma das maiores preocupações mundiais da atualidade e foco de mobilização em muitos países (BRASIL, 2018; MALTA et al., 2014). Com vistas a essa epidemia de doenças crônicas, e visando à mudança do modelo assistencial, os profissionais de educação física e os nutricionistas trabalham, em sua rotina profissional, no intuito de promover saúde sem a necessidade de tratamentos farmacológicos precoces, prevenindo o desencadeamento de agravos à saúde mediante proposta de uma alimentação saudável e a realização de exercícios físicos regulares, sendo estes ferramentas essenciais para a prevenção de doenças e manutenção da saúde.

Os campos de atuação destes profissionais rotineiramente se envolvem, estando a nutrição e a educação física por muitos momentos entrelaçadas em seus núcleos do saber, propiciando pela consulta com- partilhada, atender de maneira mais resolutiva às demandas dos indivíduos, ampliando a assistência não apenas para os conhecimentos de núcleo do saber, mas de campo: o campo da saúde pública (CAMPOS, 2000). Dentre as demandas mais frequentes trazidas pelos usuários nas consultas compartilhadas, estão a redução de peso, dislipidemia, DM, HAS e DCV.

A presença concomitante de uma ou mais doenças é frequente. Visando a atender às queixas e controlar a ação negativa dessas comorbidades na vida das pessoas, organizou-se no serviço, com o apoio de médicos e enfermeiros, uma rotina de não medicalização imediata. Assim, antes da indicação de terapias farmacológicas, os usuários eram encaminhados para o atendimento compartilhado (nutricional e de educação física).

A fim de otimizar o tempo dos indivíduos dentro do serviço, melhorar a assistência e contemplar o usuário como um todo nesta oferta de cuidado, estabeleceu-se a rotina dos atendimentos compartilhados no turno estendido (noturno). A faixa etária dos sujeitos encaminhados para os atendimentos compartilhados variava entre crianças (8 e 9 anos) e idosos (maiores de 70 anos).

Os atendimentos eram realizados pelos dois profissionais simultaneamente. Uma anamnese, constituída de perguntas sobre alimentação, atividades diárias, atividade física, hábito intestinal, trabalho, local da realização das refeições, aquisição dos alimentos, moradia e avaliação antropométrica, era realizada neste momento.

Posterior a este primeiro contato, os profissionais discutiam, juntamente com o usuário, a condição social e de saúde em que estavam inseridos, e se organizava quais seriam as intervenções possíveis de serem adotadas perante os hábitos de vida destes, para que se tivesse um desfecho positivo sobre o controle das patologias e intercorrências relatadas. Após esta fase, os usuários eram estimulados a adotar tais metas e recomendações construídas em concomitância, sendo sempre ressaltada a importância do autocuidado e da responsabilização do usuário, sendo ele o protagonista do seu cuidado.

$O$ atendimento de crianças, adultos e idosos era preferencialmente individualizado. No caso das crianças menores de oito anos de idade, os pais acompanhavam o atendimento, todavia, em alguns casos, era solicitado que os pais se ausentassem do consultório para que a criança pudesse se sentir mais à vontade para expor suas questões. As metodologias de aten- 
dimento e abordagem das situações diferenciavam-se de acordo com o público, sendo levado em consideração o nível de instrução (escolaridade) dos usuários.

A proposta dos atendimentos compartilhados faz parte do constructo da clínica ampliada, que compõe a Política Nacional de Humanização (BRASIL, 2004), que visa a qualificar o modo de fazer saúde por meio do estabelecimento de vínculo dos profissionais com os usuários, aumento do empoderamento, autonomia dos usuários pela sua saúde e o estabelecimento de um atendimento multiprofissional, podendo ser pensadas as condutas que serão adotadas de acordo com cada caso.

Por diversos momentos foram prestadas orientações e explicações sobre questões que não englobam o campo do saber dos profissionais supracitados, todavia entende-se que todo profissional da saúde integra o campo do saber em saúde, e que necessita associar, de maneira dinâmica, as demandas dos indivíduos, sendo capaz de orientar sobre saúde nas mais diversas intercorrências, e sempre que necessário direcionar o usuário a atendimento especializado. Metodologias para ampliar os conhecimentos dos profissionais foram criadas, e, hoje, momentos de educação continuada são espaços de sabedoria e troca de experiências, em que as equipes são incentivadas a ampliar sua rede de conhecimento, visando à qualificação profissional (PAGANI; ANDRADE, 2012; BRASIL, 2010; BRASIL, 2009).

Neste panorama de atenção integral, a residência multiprofissional apresenta-se com mais uma ferramenta para promover saúde de maneira a empoderar os sujeitos para o seu cuidado, com apoio de profissionais de diversas áreas buscando atender o princípio da integralidade nos serviços de saúde por intermédio da clínica ampliada. No serviço abordado, as consultas compartilhadas já estão estabelecidas dentro do fluxo do serviço, principalmente porque os profissionais de saúde já a incorporaram em suas rotinas e conseguem identificar o quão benéfico ela é para a melhoria da qualidade de vida, autonomia, diminuição das queixas e do agravamento de doenças na população.

Trabalhar de maneira conjunta, em duas ou mais áreas do saber na atenção à saúde dos usuários, faz com que os mesmos consigam entender de forma mais efetiva a importância de algumas condutas na promoção e recuperação da saúde e prevenção de agravos. O trabalho multiprofissional, na lógica da interdisciplinaridade, é uma possibilidade de ampliar a capacidade humana para compreender a realidade e os problemas que nela se apresentam, favorecendo a articulação do conhecimento de várias áreas com os seus saberes e os seus fazeres, de modo a dar mais sentido à teoria, ampliar a compreensão dos problemas de saúde e, consequentemente, melhorar a prática em saúde (OLIVEIRA et al., 2011).

A complexidade dos sujeitos pode causar estranhamento nas equipes de saúde, todavia a clínica ampliada, que embasa a lógica do atendimento compartilhado, vem para suprir estas questões, pois a combinação das especialidades em saúde irão contemplar de maneira global as demandas dos usuários, e não fragmentando-os em diagnósticos e procedimentos como se fossem objetos (BRASIL, 2009).

Esta metodologia de prática em saúde engloba o usuário na sua totalidade, sendo considerada a individualidade de cada um e todas as relações que o cercam, na busca de um cuidado em saúde que melhor se adeque as suas condições e possibilidades. O fortalecimento da APS como serviço de saúde de referência pode ser desenvolvido por todos os profissionais que compõem a rede de cuidado aos usuários, vislumbrando a promoção de condutas que mantenham a autonomia e capacidades de decisão dos usuários, fazendo com que estes se sintam parte da gestão do cuidado com igual responsabilização e importância (BRASIL, 2018).

\section{CONCLUSÕES}

A troca intensa de saberes profissionais em diversos campos, exercendo, dentro de um mesmo cenário, uma ação de reciprocidade e mutualidade, que pressupõe uma atitude diferenciada diante de um determinado problema, é o ponto-chave para uma assistência integral e resolutiva segundo os princípios e diretrizes do Sistema Único de Saúde, bem como da atenção primária em saúde. A promoção da saúde e do autocuidado, de maneira a empoderar o sujeito e colocá-lo como protagonista e responsável pela sua saúde, é o que pressupõe a APS, tendo no atendimento compartilhado um disparador de ações de mútua responsabilidade que fortalece os vínculos entre os profissionais da equipe de saúde, bem como os laços entre os profissionais de saúde e os usuários.

\section{AGRADECIMENTOS}

Ao Ministério da Saúde pela bolsa de residência, à Fundação Municipal de Saúde de Santa Rosa Fumssar - e também ao Núcleo de Ensino e Pesquisa da Fumssar - NEP.

Conflito de interesses: Declaramos não haver conflito de interesses. 


\section{REFERÊNCIAS}

ALMEIDA, J. H. H. et al. Atenção primária à saúde: enfocando as redes de atenção à saúde. Rev. Enferm. Ufpe [on-line], Recife, v. 9, n. 11, p. 9.811-9.816, nov. 2015.

ARPINI, L. S. B.; ARPINI, A. F. Integração dos campos de saúde coletiva e alimentação e nutrição num contexto de promoção da saúde relacionado às doenças crônicas não transmissíveis. Demetra, Rio de Janeiro, v. 9, n. 2, p. 451465, 2014.

BRASIL. Ministério da Saúde. HumanizaSUS: política nacional de humanização: a humanização como eixo norteador das práticas de atenção e gestão em todas as instâncias do SISTEMA ÚNICO DE SAÚDE (SUS). Brasília: Ministério da Saúde, 2004. 20p. Disponível em: <http://bvsms.saude.gov. $\mathrm{br} / \mathrm{bvs} /$ publicacoes/humanizasus_2004.pdf>. Acesso em: mar. 2017.

. Ministério da Saúde. Secretaria de Vigilância à Saúde. Secretaria de Atenção à Saúde. Diretrizes e recomendações para o cuidado integral de doenças crônicas não-transmissíveis: promoção da saúde, vigilância, prevenção e assistência. Brasília: Ministério da Saúde, 2008. 72p. Disponível em: <http://bvsms.saude.gov.br/bvs/publicacoes/ diretrizes_recomendacoes_cuidado_doencas_cronicas.pdf >. Acesso em: jun. 2017.

. Ministério da Saúde. Secretaria de Atenção à Saúde. Política Nacional de Humanização da Atenção e Gestão do SUS. Clínica ampliada e compartilhada. Brasília: Ministério da Saúde, 2009. 64p. Disponível em: <http://bvsms. saude.gov.br/bvs/publicacoes/clinica_ampliada_compartiIhada.pdf>. Acesso em: abr. 2017.

. Ministério da Saúde. Secretaria de Atenção à Saúde. Departamento de Atenção Básica. Diretrizes do Nasf: Núcleo de Apoio à Saúde da Família. Brasília: Ministério da Saúde, 2010. 152p. (Série A. Normas e Manuais Técnicos; Caderno de Atenção Básica, n. 27). Disponível em: <http://189.28.128.100/dab/docs/publicacoes/cadernos_ ab/abcad27.pdf>. Acesso em: jun. 2017.

. Ministério da Saúde. Secretaria de Atenção à Saúde Departamento de Ações Programáticas e Estratégicas. Orientações técnicas para a implementação de Linha de Cuidado para Atenção Integral à Saúde da Pessoa Idosa no
Sistema Único de Saúde - SUS [recurso eletrônico]. Brasília: Ministério da Saúde, 2018. 86p. Disponível em: <https:// www.mds.gov.br/webarquivos/publicacao/Brasil_Amigo_ Pesso_Idosa/Orientacoes_Implementacao_Linha_Cuidado_Atencaolntegral_Saude_Pessoa_Idosa_SŪS.pdf $>$. Acesso em: nov. 2018.

CAMPOS, G. W. S. Saúde pública e saúde coletiva: campo e núcleo de saberes e práticas. Ciência e Saúde Coletiva, Rio de Janeiro, v. 5, n. 2, supp. 2, p. 219-230, jan./dez. 2000.

CORRÊA, L.Q. et al. A atuação da educação física nas residências multiprofissionais em saúde. Revista Brasileira de Promoção de Saúde, Fortaleza, v. 27, n. 3, p. 428-433, jul./ set. 2014.

COSTA, J. P. et al. Resolubilidade do cuidado na atenção primária: articulação multiprofissional e rede de serviços. Saúde em Debate, Rio de Janeiro, v. 38, n. 103, p.733-743, dez. 2014.

MALTA, D. C. et al. Mortalidade por doenças crônicas não transmissíveis no Brasil e suas regiões, 2000 a 2011. Epidemiologia e Serviços de Saúde, Brasília, v. 23, n. 4, p. 599608, dez. 2014.

OLIVEIRA, E. R. A. et al. Interdisciplinaridade, trabalho em equipe e multiprofissionalismo: concepções dos acadêmicos de enfermagem. Revista Brasileira de Pesquisa em Saúde, Espírito Santo, v. 13, n. 4, p. 28-34, 2011.

PAGANI, R.; ANDRADE, L. O. M. Preceptoria de território, novas práticas e saberes na estratégia de educação permanente em saúde da família: o estudo do caso de Sobral, CE. Saúde e Sociedade, São Paulo, v. 21, supl. 1, p. 94-106, maio 2012.

SOUZA, E. C. F. et al. Acesso e acolhimento na atenção básica: uma análise da percepção dos usuários e profissionais de saúde. Cadernos de Saúde Pública, Rio de Janeiro, v. 24, supl. 1, p. 100-110, 2008.

TOMASI, A. R. P.; RIZZOTTO, M. L. F. Análise da distribuição e composição profissional dos Núcleos de Apoio à Saúde da Família no Paraná. Saúde em Debate, Rio de Janeiro, v. 37, n. 98, p. 427-36, jul./set. 2013.

VIEIRA, D. C. D. et al. Percepções de usuários da Estratégia de Saúde da Família sobre saúde, prevenção e autocuidado. Revista de Enfermagem UFPE [on-line], Recife, v. 10, n. 2, p. 413-418, fev. 2016. 